\title{
Crecimiento y composición bioquímica de Thalassiosira pseudonana (Thalassiosirales: Thalassiosiraceae) bajo cultivo semi-continuo en diferentes medios y niveles de irradiancias
}

\author{
Aleikar Vásquez-Suárez ${ }^{1}$, Miguel Guevara ${ }^{1}$, Mayelys González ${ }^{2}$, Roraysi Cortez ${ }^{1}$ \\ \& Bertha Arredondo-Vega ${ }^{3}$ \\ 1. Universidad de Oriente, Instituto Oceanográfico de Venezuela, Departamento de Biología Pesquera, Laboratorio de \\ Acuicultura, Extensión Plancton. C. P. 6101. Cumaná, estado Sucre, Venezuela; valeikar@yahoo.es, \\ miguevara2003@yahoo.es, roraysi@yahoo.com \\ 2. Postgrado en Biología Aplicada, Casa N 13, Cerro del Medio, Universidad de Oriente, Núcleo de Sucre, C. P. 6101. \\ Cumaná, estado Sucre, Venezuela; mayelysg@hotmail.com \\ 3. Laboratorio de Biotecnología de Microalgas, Centro de Investigaciones Biológicas del Noroeste (CIBNOR), Apdo. \\ postal 128, La Paz, Baja California Sur, C. P. 23000, México; kittybcs2005@yahoo.com.mx
}

Recibido 02-VII-2012. Corregido 10-I-2013. Aceptado 08-II-2013.

\begin{abstract}
Growth and biochemical composition of Thalassiosira pseudonana (Thalassiosirales: Thalassiosiraceae) cultivated in semicontinuous system at different culture media and irradiances. Thalassiosira pseudonana is a marine Bacillariophyta commonly used as live feed in mariculture. The growth rate and biochemical composition of microalgae are highly influenced by environmental factors such as, irradiance and nutrient availability. The aim of this study was to investigate the influence of three irradiances $(60$, 120 and $180 \mu \mathrm{E} / \mathrm{m}^{2} . \mathrm{s}$ ) and two culture media (Algal and Humus) on growth and biochemical composition of this diatom. The microalga was grown semicontinuously at a daily renewal rate of fresh media of $30 \%, 37 \%$ o salinity, $25 \pm 1^{\circ} \mathrm{C}$ and constant aeration $(200 \mathrm{~mL} / \mathrm{min})$. The cell densities (cel $\left./ \mathrm{mL}\right)$ and contents of protein, lipid, carbohydrate, chlorophyll $a$, total carotenoids, and fatty acids, showed significant differences $(\mathrm{p}<0.05)$ between treatments. During steady-state phase, the maximal cell density, and lipid and carbohydrate contents were of $4.62 \times 10^{6} \mathrm{cel} / \mathrm{mL}, 20.3 \pm 2.28 \%$ and $16.6 \pm 2.43 \%$, respectively, and were achieved in Humus medium at $180 \mu \mathrm{E} /$ $\mathrm{m}^{2}$.s. Moreover, highest protein contents $(45.0 \pm 5.05 \%)$ and total carotenoids $(0.5 \pm 0.01 \%)$ were obtained in Algal medium at $180 \mu \mathrm{E} / \mathrm{m}^{2}$.s. Chlorophyll $a(0.93 \pm 0.04 \%)$ was higher at low irradiances in Algal medium. In both media, the fatty acids unsaturation degree was lower with increasing irradiance, being eicosapentaenoic acid, 20:5 n-3 (EPA) most represented (6.20\%) in Algal medium at $60 \mu \mathrm{E} / \mathrm{m}^{2}$.s. This strain of T. pseudonana showed multiple physiological responses to changes in culture conditions, and may be cultivated with an alternative medium, which reduced the operating costs and allowed a high nutritional biomass production value for animals under culture. Rev. Biol. Trop. 61 (3): 1003-1013. Epub 2013 September 01.
\end{abstract}

Key words: Thalassiosira pseudonana, tropical aquaculture, fatty acids, native, semicontinuous culture.

La disponibilidad de nutrientes y luz son los principales factores que influyen directa y separadamente sobre la fisiología del fitoplancton. Mientras los nutrientes son necesarios para la síntesis de biomasa, la irradiancia y calidad de luz determina la cantidad de energía disponible para actividades metabólicas. Las alteraciones fisiológicas que ocurren en el metabolismo celular, bajo condiciones limitantes para el crecimiento de las microalgas, son diferentes y dependen, principalmente, de la especie, de la cepa y de los factores ambientales involucrados (Shaw et al. 1989). Las microalgas pueden adaptarse a cambios de irradiancia, originando 
variaciones considerables en su composición bioquímica y metabolismo (Sánchez-Saavedra \& Voltolina 2002).

Los pigmentos fotosintéticos en su doble rol como componentes de biomasa y como sistema productor de energía son afectados cualitativa y cuantitativamente por cambios en la irradiancia de los cultivos. Las células responden de tal manera que modifican sus estructuras físicas y químicas a fin de optimizar las concentraciones pigmentarias que permitan el funcionamiento de la maquinaria fotosintética. Sin embargo, cuando la deficiencia de nutrientes representa un factor limitante, los pigmentos no están directamente relacionados con el proceso y sus concentraciones dependen principalmente de los precursores necesarios para su síntesis (Rosen \& Lowe 1984). Estas cualidades de fotoadaptación microalgal han sido aprovechadas por diversos investigadores con el propósito de incrementar la producción de metabolitos de interés biotecnológico (Sandnes et al. 2005, Solovchenko et al. 2008, Vílchez et al. 2011) o para mejorar la calidad nutricional de microalgas que son utilizadas comúnmente en acuicultura (Meseck et al. 2005, Priyadarshani et al. 2012).

Por otro lado, las diatomeas planctónicas representan uno de los principales grupos autotróficos en ecosistemas marinos y fuente importante de alimento para sustento de la acuicultura. Diatomeas del género Thalassiosira son frecuentemente utilizadas como alimento de moluscos (Kiatmetha et al. 2011) debido a su alto contenido de ácidos grasos poliinsaturados (AGPs); sin embargo, las cantidades producidas por esta microalga, no satisfacen las necesidades del sector acuícola, que evidencia un vertiginoso crecimiento a nivel mundial (Mischke \& Zimba 2004), haciéndose necesario desarrollar sistemas de cultivos masivos con calidad bioquímica y/o valor nutricional estable, además de mínimos riesgos de ser contaminados. Adicionalmente, se han probado numerosos medios de cultivo alternativos (Valenzuela-Espinoza et al. 2005, Guzmán-Murillo et al. 2007), debido a los considerables costos que implica la producción de alimento vivo.

En la búsqueda de mejores esquemas de manejo que permitan predecir el valor nutricional de microalgas bajo condiciones controladas, se han ensayado diferentes estrategias entre las que destacan la manipulación de variables ambientales y el tipo o sistema de cultivo. El sistema discontinuo o batch, se caracteriza porque no hay adición o eliminación de nutrientes posterior a la inoculación, comprometiendo la estabilidad de la composición bioquímica microalgal a consecuencia de la disminución de nutrientes con el avance de la edad/fase del cultivo (Zhukova 2004). En el sistema semicontinuo, existe reposición diaria de nutrientes a una tasa de dilución constante, lo que genera mayor estabilidad en las condiciones de cultivo, razón que motivó a seleccionar este último.

A fin de contribuir con el conocimiento de las respuestas fisiológicas de la cepa T. pseudonana, aislada de aguas tropicales, ante cambios en las condiciones de su entorno, se propuso evaluar el efecto combinado de la irradiancia y del medio de cultivo sobre su crecimiento y composición bioquímica; buscando además, incrementar la variedad de especies microalgales, con calidad nutricional, que puedan servir de alimento a larvas de organismos superiores en la cadena trófica que son producidos en criaderos de países tropicales.

\section{MATERIALES Y MÉTODOS}

Microorganismo seleccionado y condiciones de cultivo: La cepa (CCPUDO-39) de $T$. pseudonana, aislada de la costa oeste en la Península de Araya, estado Sucre, (10³7'03" $\left.\mathrm{N}-64^{\circ} 07^{\prime} 45^{\prime \prime} \mathrm{W}\right)$, Venezuela y depositada en la Colección de Cultivos Planctónicos de la Universidad de Oriente (CCPUDO-39) fue cultivada, por triplicado en cada una de las condiciones probadas, bajo régimen semicontinuo, durante 15 días, en ambiente controlado, con tasa de renovación diaria equivalente al $30 \%$ del cultivo. La temperatura promedio fue $25 \pm 1^{\circ} \mathrm{C}$ y fotoperiodo luz:oscuridad de 12:12. Se utilizaron matraces con capacidad de $250 \mathrm{~mL}$ 
(equipados con mangueras plásticas y capilares para una aireación constante de $200 \mathrm{~mL} / \mathrm{min}$ ). El agua de mar (37PSU) fue filtrada con papel Whatman $\mathrm{GF} / \mathrm{C}$ de $1.2 \mu \mathrm{m}$ en tamaño de poro y autoclavada a $120^{\circ} \mathrm{C} / 15$ psi de presión por $15 \mathrm{~min}$. Parte del diseño experimental consistió en probar tres irradiancias $(60,120 \mathrm{y} 180 \mu \mathrm{E} /$ $\mathrm{m}^{2}$.s) en dos medios de cultivo, uno denominado Algal, $\mathrm{pH}=7.97 \pm 0.05$ (Fábregas et al. 1984) de uso frecuente en acuicultura y el otro, un fertilizante comercial de bajo costo (Humus líquido $^{\circledR}$ a $\mathrm{pH} 7.6 \pm 0.05$ ); la constitución de estos medios, de acuerdo a sus diseñadores o fabricantes, se listan en el cuadro 1. Ambos medios de cultivo se prepararon a una concentración de $4 \mathrm{mmoles} / \mathrm{L}$ de nitrato.

\section{CUADRO 1}

Composición de los medios de cultivo utilizados en el crecimiento de Thalassiosira pseudonana bajo sistema semicontinuo según fabricante

TABLE 1

Composition of the culture media used for Thalassiosira pseudonana growth under semicontinuous system after product label

\begin{tabular}{lclc}
\multicolumn{1}{c}{ Medio Algal } & $(\mathrm{mg} / \mathrm{L})$ & \multicolumn{1}{c}{ Medio Humus } & $(\mathrm{mg} / \mathrm{L})$ \\
$\mathrm{ZnCl} 2$ & 13.6 & Ácidos húmicos & 330 \\
$\mathrm{MnCl} 2.4 \mathrm{H} 2 \mathrm{O}$ & 19.79 & Nitrógeno amoniacal & 21 \\
$\mathrm{NaMoO} 4.2 \mathrm{H} 2 \mathrm{O}$ & 24.2 & Nitrógeno en Nitrato & 4 \\
$\mathrm{CoCl} 3$ & 1.65 & Nitrógeno Orgánico & 20 \\
$\mathrm{CuSO} 4.5 \mathrm{H} 2 \mathrm{O}$ & 2.49 & Fósforo & 34 \\
EDTA-Na & 978 & Potasio & 45 \\
FeC6H5O7 & 670 & Carbonato orgánico & 350 \\
$\mathrm{KNO} 3$ & 17000 Calcio & 40 \\
NaH2PO4.2H2O & 1560 Cobalto & 15 \\
Tiamina & 3.5 & Cobre & 190 \\
Biotina & 0.5 & Zinc & 125 \\
Cianocobalamina & 0.3 & Manganeso & 650 \\
& & Magnesio & 50 \\
& & Hierro & 100 \\
& & Boro & 40 \\
\hline
\end{tabular}

Cultivos de la diatomea, previamente aclimatados durante 10 días y en fase de crecimiento exponencial, sirvieron para inocular $\left(2.0 \times 10^{5} \mathrm{cel} / \mathrm{mL}\right)$ los matraces de vidrio contentivos del agua de mar con las condiciones arriba señaladas. Cuando los cultivos alcanzaron la fase de crecimiento exponencial, se procedió a realizar recambios diarios del 30\% de su volumen total, es decir, el retiro del $30 \%$ del volumen total del cultivo, seguido de la reposición del mismo volumen, con su respectivo medio fresco.

Crecimiento poblacional: Desde el inicio de los recambios, y cada 24 horas, se tomaron muestras $(1 \mathrm{~mL})$, por triplicado de cada tratamiento, y se fijaron con $50 \mu \mathrm{L}$ de una solución de lugol al 10\% para realizar el recuento celular por microscopia de luz, utilizando un hematocitómetro Neubaüer de $0.01 \mathrm{~mm}$ de profundidad. Con los datos obtenidos se elaboraron las curvas de crecimiento y a partir de éstas se determinaron las tasas instantánea de crecimiento, siguiendo las recomendaciones en Madigan et al. (1999).

Determinación de la composición bioquímica: Cuando los cultivos alcanzaron la fase de estabilización (caracterizada por no observar variaciones en las tasas de crecimiento), se tomaron muestras de $5 \mathrm{~mL}$, por triplicado en cada una de las variables estudiadas, las cuales se centrifugaron. El pellet celular obtenido se utilizó para realizar análisis del contenido de proteínas (Lowry et al. 1951), lípidos (Bligh \& Dyer 1959, Marsh \& Weinstein 1966) y carbohidratos (Dubois et al. 1956) totales, utilizando como estándares albúmina bovina, tripalmitina y D-glucosa, respectivamente. Para determinar el perfil de ácidos grasos, los extractos lipídicos, por triplicado, fueron transesterificados, en ésteres metílicos siguiendo las recomendaciones de Sato \& Murata (1988). Los ésteres metílicos de ácidos grasos previamente obtenidos fueron separados y cuantificados utilizando un cromatógrafo de gases-espectrómetro de masas (GC-MS) Hewlett Packard Series G1800B, acondicionado con una columna Omegawax TM 250 de sílica fundida (Supelco) de 30x0.25mm de diámetro interno. Los ácidos grasos presentes en las muestras se identificaron mediante la comparación de los espectros de masas con los 
espectros contenidos en la biblioteca de espectros de masas NIST98, NBS75K y una biblioteca creada con 28 estándares de metil-ésteres de ácidos grasos (Sigma Chemical Company). Adicionalmente, se confirmó la identificación de los ácidos grasos mediante la comparación de los tiempos de retención de las muestras con los registrados para una mezcla comercial de metil-ésteres de ácidos grasos poliinsaturados (Sigma). También se determinó el contenido celular de clorofila $a$ y carotenoides totales siguiendo la metodología en Strickland \& Parsons (1972).

Los datos de crecimiento y composición bioquímica se analizaron a través de análisis de varianza de dos vías, considerando los niveles de irradiancia y los medios de cultivo como factores. Los datos fueron transformados a logaritmos de base diez, +10 , para obtener homogeneidad de varianzas y distribución normal de los datos, siguiendo recomendaciones en Sokal \& Rohlf (1995), además se aplicaron en casos necesarios las pruebas a posteriori de Scheffè.

\section{RESULTADOS}

Crecimiento poblacional: En ambos medios de cultivo, T. pseudonana inició su fase de estabilización al noveno día de recambio en las dos menores irradiancias (60 y $120 \mu \mathrm{E} /$ $\mathrm{m}^{2}$.s) y al décimo primer día de recambio a $180 \mu \mathrm{E} / \mathrm{m}^{2}$.s (Fig. 1A, 1B y 1C). Las irradiancias, los medios de cultivo y la interacción de ambos influyeron significativamente $(\mathrm{p}<0.05)$ sobre el crecimiento de la diatomea, en los que se observó mayores densidades celulares $\left(4.6 \pm 0.37 \times 10^{6} \mathrm{cel} / \mathrm{mL}\right)$ a $180 \mu \mathrm{E} / \mathrm{m}^{2} . \mathrm{s}$ en Humus; sin embargo, en este mismo medio pero a irradiancias de 60 y $120 \mu \mathrm{E} / \mathrm{m}^{2}$.s, se registraron las menores densidades celulares, con valores de $1.9 \pm 0.24$ y $1.2 \pm 0.16 \times 10^{6} \mathrm{cel} /$ $\mathrm{mL}$, respectivamente. Las tasas instantáneas de crecimiento variaron entre 0.45 y $0.56 \mathrm{div} /$ día sin mostrar diferencias significativas $(\mathrm{p}>0.05)$ entre las distintas condiciones de cultivo.
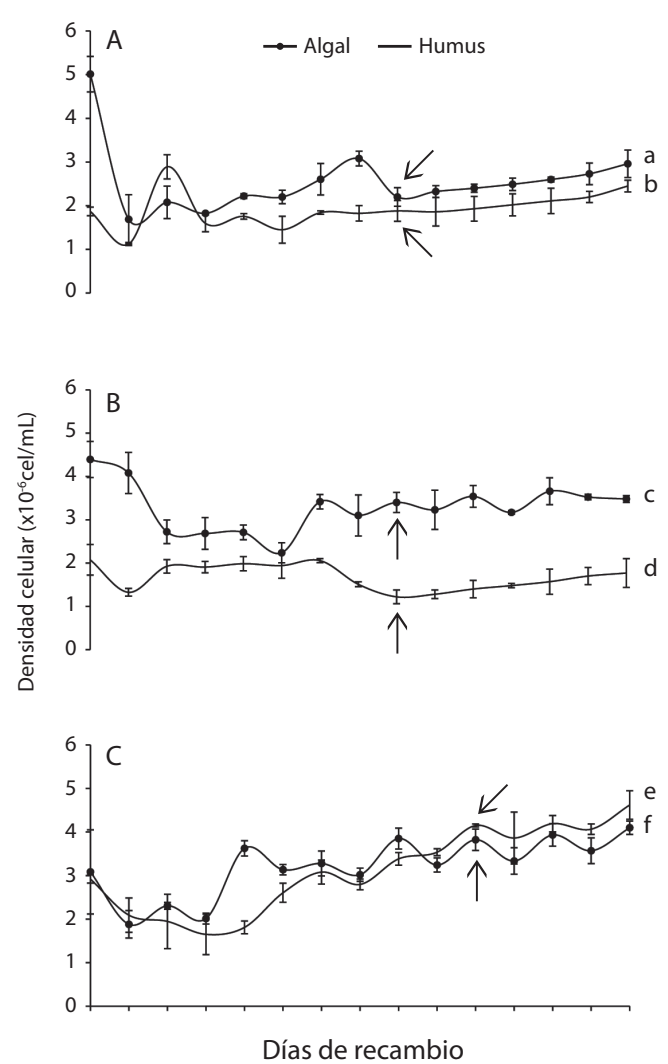

Fig. 1. Crecimiento (Media $\pm \mathrm{DS})$ de Thalassiosira pseudonana cultivada en sistema semicontinuo a $60 \mu \mathrm{E} /$ $\mathrm{m}^{2}$.s (A), $120 \mu \mathrm{E} / \mathrm{m}^{2}$.s (B) y $180 \mu \mathrm{E} / \mathrm{m}^{2}$.s (C) en medio Algal y Humus. Las puntas de flechas indican el inicio de la fase de estabilización. Letras diferentes representan grupos estadísticamente distintos $(\mathrm{p}<0.05)$.

Fig. 1. Growth (means \pm DS) of Thalassiosira pseudonana cultured semicontinuously at $60 \mu \mathrm{E} / \mathrm{m}^{2}$.s (A), $120 \mu \mathrm{E} / \mathrm{m}^{2}$.s (B) y $180 \mu \mathrm{E} / \mathrm{m}^{2} \mathrm{~s}$ (C) in Algal and Humus media. The arrowheads indicate beginning steady-state phase. All values are average \pm standard deviation $(n=3)$. Same letters indicate no significant differences between averages (ANOVA, Scheffès F-test; $\mathrm{p}>0.05$ ).

Bioquímica: Se observaron diferencias en el contenido de proteínas totales $(\mathrm{p}<0.05)$ cuando la diatomea fue cultivada en los dos medios a las diferentes irradiancias. El máximo contenido proteico $(44.9 \pm 5.05 \%$, Fig. 2A) se obtuvo a la mayor irradiancia evaluada y en el medio Algal. El contenido de lípidos totales de T. pseudonana (Fig. 2B) mostró diferencias 
significativas $(\mathrm{p}<0.05)$ entre los diferentes medios e irradiancias. Esta macromolécula alcanzó su mayor contenido $(20.3 \pm 2.28 \%)$ en Humus a $180 \mu \mathrm{E} / \mathrm{m}^{2} . \mathrm{s}$; mientras que el menor porcentaje $(7.8 \pm 1.62 \%)$ se observó en medio Algal a $60 \mu \mathrm{E} / \mathrm{m}^{2}$.s. Se determinó interacción entre los factores $(p<0.05)$, el cual deja en evidencia, un efecto sinérgico entre el medio Humus y la irradiancia, en función de una mayor concentración de lípidos.

Los carbohidratos totales (Fig. 2C) sólo mostraron diferencias significativas $(\mathrm{p}<0.05)$ entre las irradiancias; obteniéndose las mayores concentraciones de $16.7 \pm 2.43$ y $15.7 \pm 0.74 \%$ a 180 y $120 \mu \mathrm{E} / \mathrm{m}^{2} . \mathrm{s}$, respectivamente, lo cual evidencia que ambas irradiancias ejercen efectos similares sobre la acumulación de carbohidratos en esta microalga.

Los AGPs representaron entre el 8.02 y $17.13 \%$ del total de ácidos grasos en T. pseudonana (Cuadro 2), encontrándose diferencias significativas $(p<0.05)$ en las concentraciones de éstos, bajo las diferentes condiciones de cultivo ensayadas. El principal ácido graso poliinsaturado sintetizado fue el 20:5 n-3 (AEP), con mayores concentraciones de éste en medio Algal a 60 y $120 \mu \mathrm{E} / \mathrm{m}^{2}$.s (6.2 y $6.03 \%$ respectivamente), mientras que en medio Humus se observó disminución en la biosíntesis de AEP a medida que se incrementó la irradiancia de los cultivos. El 20:4 n-6 (AA) fue sintetizado en mayor porcentaje en medio Algal a $180 \mu \mathrm{E} /$ $\mathrm{m}^{2}$.s (2.16\%), mientras que de los AGPs con $\mathrm{C}_{16}$, el más abundante fue el 16:3, con porcentajes máximos a la menor irradiancia en medio Algal (3.78\%). Se detectaron cantidades relativamente pequeñas (entre 0.22 y $0.3 \%$ ) de ácido docosahexaenoico, 22:6 n-3 (ADH), observándose un incremento de su concentración a medida que se incrementaba la irradiancia. Los contenidos totales de ácidos grasos saturados y monoinsaturados fueron observados en proporciones similares en Algal a las diferentes irradiancias, mientras que en Humus se observó mayor concentración de los saturados. El 16:1 n-7 fue el componente representativo

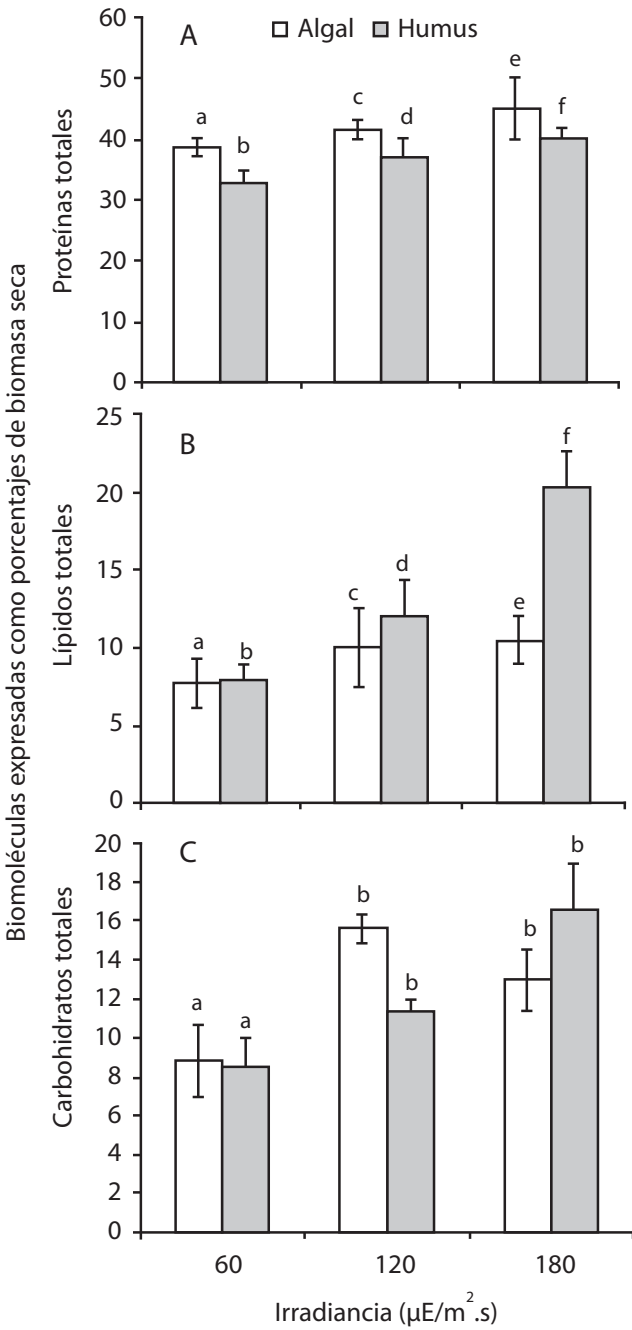

Fig. 2. Contenido de proteínas (A), lípidos (B) y carbohidratos totales (C), (\% de biomasa seca) de Thalassiosira pseudonana cultivada en medio Algal y Humus a tres irradiancias $\left(60,120\right.$ y $\left.180 \mu \mathrm{E} / \mathrm{m}^{2} . \mathrm{s}\right)$ bajo sistema semi-continuo. Todos los valores representan el promedio \pm desviación estándar $(n=3)$. Letras iguales representan grupos estadísticamente iguales $(\mathrm{p}>0.05)$.

Fig. 2. Total contents (as \% dry weight) of protein (A), lipids (B) and carbohydrates (C) of Thalassiosira pseudonana grown in Algal and Humus media at three irradiances $\left(60,120\right.$ y $\left.180 \mu \mathrm{E} / \mathrm{m}^{2} . \mathrm{s}\right)$ under semicontinuous system. All values are average \pm standard deviation $(n=3)$. Same letters indicate no significant differences between averages (ANOVA, Scheffès F-test; $\mathrm{p}>0.05$ ). 
CUADRO 2

Perfil de ácidos grasos de Thalassiosira pseudonana (\% del total de ácidos grasos) cultivada en medio Algal y Humus a tres irradiancias bajo sistema semi-continuo

TABLE 2

Fatty acids profile of Thalassiosira pseudonana ( $\%$ of total fatty acids) grown in Algal and Humus media at three irradiances under semicontinuous system

\begin{tabular}{|c|c|c|c|c|c|c|}
\hline & \multicolumn{3}{|c|}{ Medio Algal } & \multicolumn{3}{|c|}{ Medio Humus } \\
\hline $\begin{array}{l}\text { Ácido Graso } \\
\text { (\% del total) }\end{array}$ & 60 & 120 & 180 & 60 & 120 & 180 \\
\hline $14: 0$ & 10.45 & 9.58 & 14.94 & 9.37 & 18.00 & 12.47 \\
\hline $15: 0$ & 0.66 & 0.97 & 0.79 & 2.95 & 1.64 & 0.82 \\
\hline $16: 0$ & 18.79 & 31.12 & 23.57 & 31.37 & 26.85 & 29.50 \\
\hline $16: 0$ ante iso & 1.97 & 0.26 & 0.27 & 0.56 & 0.67 & 0.33 \\
\hline $17: 0$ & 0.27 & 0.18 & 0.83 & 0.26 & 0.57 & 0.93 \\
\hline 18:0 & 10.44 & 4.53 & 12.73 & 9.71 & 8.30 & 16.18 \\
\hline $20: 0$ & 0.33 & 0.16 & 0.66 & 0.43 & 0.21 & 2.33 \\
\hline 22:0 & 0.46 & 0.35 & 0.45 & 0.40 & 0.43 & 0.37 \\
\hline $24: 0$ & 0.21 & 0.19 & 0.15 & 0.29 & 0.16 & 0.10 \\
\hline$\sum$ Saturados & 43.58 & 47.34 & 54.39 & 55.34 & 56.83 & 63.03 \\
\hline $16: 1 \mathrm{n}-9$ & 3.69 & 4.95 & 3.15 & 2.59 & 0.77 & 1.14 \\
\hline $16: 1 \mathrm{n}-7$ & 16.53 & 16.05 & 14.86 & 17.10 & 20.46 & 15.06 \\
\hline $16: 1 \mathrm{n}-5$ & 0.13 & 1.34 & 0.25 & 0.16 & 0.25 & 0.25 \\
\hline $16: 1$ & 1.02 & 0.83 & 0.28 & 0.44 & 0.18 & 0.16 \\
\hline $18: 1 \mathrm{n}-9$ & 13.21 & 12.52 & 9.36 & 9.98 & 7.33 & 9.35 \\
\hline $18: 1 \mathrm{n}-7$ & 4.71 & 4.73 & 3.91 & 3.58 & 4.48 & 2.99 \\
\hline$\sum$ Monoinsaturados & 39.29 & 40.42 & 31.81 & 33.85 & 33.47 & 28.95 \\
\hline $16: 2 n-6$ & $1.42^{\mathrm{a}}$ & $0.49^{c}$ & $0.37^{\mathrm{c}}$ & $0.67^{\mathrm{b}}$ & $0.64^{\mathrm{b}}$ & $0.33^{\mathrm{c}}$ \\
\hline $16: 2 n-4$ & $2.73^{\mathrm{a}}$ & $0.86^{\mathrm{c}}$ & $1.17^{\mathrm{b}}$ & $0.99^{c}$ & $1.33^{\mathrm{b}}$ & $0.67^{\mathrm{d}}$ \\
\hline $16: 3$ & $3.78^{\mathrm{a}}$ & $1.13^{\mathrm{c}}$ & $1.46^{\mathrm{c}}$ & $2.25^{\mathrm{b}}$ & $1.70^{c}$ & $0.71^{\mathrm{d}}$ \\
\hline $18: 2 n-6$ & $0.72^{\mathrm{b}}$ & $1.18^{\mathrm{a}}$ & $1.10^{\mathrm{a}}$ & $0.74^{\mathrm{b}}$ & $0.82^{\mathrm{b}}$ & $0.91^{b}$ \\
\hline $18: 3 n-6$ & $0.53^{\mathrm{b}}$ & $0.97^{\mathrm{a}}$ & $1.09^{\mathrm{a}}$ & $0.12^{\mathrm{c}}$ & $0.69^{\mathrm{b}}$ & $0.63^{b}$ \\
\hline $18: 4 n-3$ & nd & nd & $0.65^{\mathrm{a}}$ & nd & nd & $0.66^{\mathrm{a}}$ \\
\hline 20:4 n-6 AA & $1.52^{\mathrm{b}}$ & $1.36^{\mathrm{c}}$ & $2.16^{\mathrm{a}}$ & $1.34^{\mathrm{c}}$ & $1.39^{\mathrm{c}}$ & $1.38^{\mathrm{c}}$ \\
\hline 20:5 n-3 AEP & $6.20^{\mathrm{a}}$ & $6.03^{\mathrm{a}}$ & $5.56^{\mathrm{a}}$ & $3.58^{b}$ & $2.87^{\mathrm{b}}$ & $2.43^{\mathrm{c}}$ \\
\hline $22: 6$ n-3 ADH & $0.23^{\mathrm{a}}$ & $0.22^{\mathrm{a}}$ & $0.24^{\mathrm{a}}$ & $0.25^{\mathrm{a}}$ & $0.26^{\mathrm{a}}$ & $0.30^{\mathrm{a}}$ \\
\hline$\sum$ Polinsaturados & $17.13^{\mathrm{a}}$ & $12.24^{\mathrm{b}}$ & $13.8^{\mathrm{b}}$ & $9.94^{\mathrm{b}}$ & $9.70^{\mathrm{b}}$ & $8.02^{\mathrm{b}}$ \\
\hline
\end{tabular}

Letras iguales en la misma fila indican grupos estadísticamente iguales $(\mathrm{p}>0.05)$.

Superíndices diferentes en una fila, representan grupos estadísticamente distintos ( $\mathrm{p}<0.05)$, nd= No detectado.

Equal superscript in same row indicates statistically equal groups $(\mathrm{p}>0.05)$

del grupo de los ácidos grasos monoinsaturados (AGMs) con valores máximos de $16.53 \%$ en Algal a $60 \mu \mathrm{E} / \mathrm{m}^{2}$.s y $20.46 \%$ en Humus a $120 \mu \mathrm{E} / \mathrm{m}^{2}$.s, seguido del $18: 1 \mathrm{n}-9$, el cual fue mayoritariamente acumulado a $60 \mu \mathrm{E} / \mathrm{m}^{2} . \mathrm{s}$ tanto en Humus como en Algal con porcentajes de $9.98 \%$ y $13.21 \%$, respectivamente. Dentro del grupo de los ácidos grasos saturados (AGSs) se observó como predominante al 16:0 con porcentajes entre $18.79 \%$ y $31.37 \%$. El 14:0 mostró los máximos valores a $60 \mu \mathrm{E} / \mathrm{m}^{2}$.s en Humus. También se evidenció mayor contenido del 18:0 cuando la microalga fue cultivada a $180 \mu \mathrm{E} / \mathrm{m}^{2} . \mathrm{s}$, tanto en Algal (12.73\%) como en Humus (16.18\%).

El nivel de irradiancia y los medios de cultivo influenciaron la composición pigmentaria. En Algal, el contenido de clorofila $a$ fue significativamente $(\mathrm{p}<0.05)$ más alto $(0.9 \pm 0.04 \%)$ durante la exposición a menor intensidad luminosa, mientras que los carotenoides totales mostraron una tendencia inversa a la clorofila $a$ (Cuadro 3), siendo significativamente más altos a la mayor irradiancia en ambos medios de cultivo, obteniéndose las máximas concentraciones $(0.5 \% \pm 0.01)$ en Algal.

CUADRO 3

Promedio \pm Desviación estándar $(\mathrm{n}=3)$

del contenido de clorofila $a$ y carotenos totales en Thalassiosira pseudonana cultivada con medio Algal y Humus a tres irradiancias bajo sistema semi-continuo

TABLE 3

Mean \pm standard deviation ( $\mathrm{n}=3$ ) of chlorophyll $a$ and total carotene content in Thalassiosira pseudonana grown in Humus and Algal media at three irradiances under semicontinuous system

\begin{tabular}{cccc}
$\begin{array}{c}\text { Irradiancia } \\
\left(\mu \mathrm{E} / \mathrm{m}^{2} . \mathrm{s}\right)\end{array}$ & $\begin{array}{c}\text { Medio de } \\
\text { Cultivo }\end{array}$ & Clorofila $a$ & $\begin{array}{c}\text { Carotenos } \\
\text { totales }\end{array}$ \\
60 & Algal & $0.933 \pm 0.04^{\mathrm{f}}$ & $0.242 \pm 0.01^{\mathrm{b}}$ \\
& Humus & $0.561 \pm 0.03^{\mathrm{d}}$ & $0.192 \pm 0.02^{\mathrm{a}}$ \\
\multirow{2}{*}{120} & Algal & $0.592 \pm 0.03^{\mathrm{e}}$ & $0.368 \pm 0.01^{\mathrm{e}}$ \\
& Humus & $0.420 \pm 0.02^{\mathrm{b}}$ & $0.291 \pm 0.02^{\mathrm{c}}$ \\
& Algal & $0.467 \pm 0.02^{\mathrm{c}}$ & $0.448 \pm 0.01^{\mathrm{f}}$ \\
& Humus & $0.285 \pm 0.01^{\mathrm{a}}$ & $0.318 \pm 0.02^{\mathrm{d}}$ \\
\hline
\end{tabular}

Los valores se encuentran expresados como porcentajes de biomasa seca.

Letras iguales en la misma columna indican grupos estadísticamente similares ( $\mathrm{p}>0.05)$.

Equal superscript in same column indicates statistically equal groups $(\mathrm{p}>0.05)$. 


\section{DISCUSIÓN}

Los cambios en los parámetros físicoquímicos de los cultivos microalgales pueden modificar la cinética de crecimiento y el valor nutricional de estos microorganismos, por lo que la producción de biomasa, contenido pigmentario, proteínas, carbohidratos y lípidos entre otros, son parámetros útiles para medir la productividad microalgal así como el estado fisiológico de las células. Los resultados obtenidos muestran que la cepa CCPUDO-39 de $T$. pseudonana bajo estudio, puede mantener una producción diaria de biomasa, con una composición bioquímica estable, durante la fase de equilibrio del régimen semicontinuo, principalmente a $180 \mu \mathrm{E} / \mathrm{m}^{2}$.s en ambos medios de cultivo. La estabilidad en las concentraciones de biomoléculas y en la densidad celular puede atribuirse a la renovación de nutrientes, lo cual promueve en los cultivos una fisiología similar a la registrada durante la fase exponencial de cultivos discontinuos (Fábregas et al. 1995).

Para maximizar la productividad celular en cultivos semicontinuos es necesario establecer inicialmente las condiciones idóneas para cada especie, y la tasa de renovación representa un factor clave en dichos sistemas de cultivo (Fábregas et al. 2000). Algunos ensayos realizados previamente, demostraron que la tasa de renovación utilizada en esta investigación era satisfactoria, coincidiendo con lo expuesto por Otero et al. (1997), lo cual permitió obtener durante la fase de estabilización en ambos medios de cultivo a $180 \mu \mathrm{E} / \mathrm{m}^{2} . \mathrm{s}$, densidades celulares superiores y tasas específicas de crecimiento similares a las reportadas por Mansour et al. (2005). A pesar de que el crecimiento de T. pseudonana en Humus a 60 y $120 \mu \mathrm{E} / \mathrm{m}^{2}$.s fue bajo, el efecto combinado de éste, con el incremento de la irradiancia permitió la mayor producción de biomasa.

De acuerdo a los resultados obtenidos, el medio Algal fue superior comparado con Humus en variables como contenido de proteínas, producción de algunos ácidos grasos polinsaturados de importancia nutricional y los pigmentos analizados, mientras que el medio
Humus, solo permitió la mayor producción de lípidos totales. Al observar las diferentes variables estudiadas y compararlas entre medios de cultivo, es posible inferir sobre la deficiencia de uno o varios nutrientes en Humus, entre ellos, los tipos y proporciones de las distintas fuentes nitrogenadas. Se ha demostrado que el nitrato como fuente de nitrógeno, ofrece mejores beneficios en cultivos intensivos que el nitrógeno amoniacal (Chaneva et al. 2007). Young \& Beardall (2003) mencionan sobre los cambios que sufren células microalgales en la fluorescencia de la clorofila por limitación de nitrato o adición de amonio a los cultivos; comportamiento que se ha interpretado como interacciones entre la fotosíntesis y asimilación de nitrógeno. Por su parte, Solovchenko et al. (2008) establecen que cultivos microalgales deficientes de nitrógeno, son más fotosensibles, posiblemente atribuido a limitaciones en la síntesis de proteínas y clorofila, lo que perjudica el funcionamiento del aparato fotosintético.

Además, también están las diferencias en las concentraciones de fósforo entre ambos medios, siendo más abundante en Algal; limitación de este elemento, causa disminución en la eficiencia del transporte de electrones y con ello, daños a la maquinaria fotosintética dificultando la captación de luz por disminución en la concentraciones celulares de pigmentos (Roberts et al. 2008), los cuales, a pesar de no poseer fósforo en su estructura, su síntesis requiere de este elemento (Hou et al. 2007). Esto podría explicar las diferencias encontradas en el contenido de proteínas, lípidos, carbohidratos, pigmentos y biomasa de T. pseudonana cultivada en ambos medios y que ha sido demostrado en Thalassiosira weissflogii (Liu et al. 2011).

En otro orden de ideas, la influencia de los niveles de irradiancia en interacción con las diferentes fuentes y concentraciones de nutrientes, ejercen un marcado efecto sobre la composición bioquímica microalgal, ya que estos microorganismos dependen de la disponibilidad de luz para la fijación de carbono, así como la asimilación de nitrógeno y fósforo necesarios en la producción de energía (Wood 
et al. 1999) que es aprovechada para crecimiento y síntesis proteica, cuando son óptimos los niveles de nutrientes e irradiancia en los cultivos. El incremento en el contenido celular de proteínas por efecto de la irradiancia evidenciado en T. pseudonana cultivada en Algal, también ha sido observado en Tetraselmis tetrathele (De la Peña \& Villegas 2005), Navicula incerta (Uriarte et al. 2006) y Chlorella vulgaris (Seyfabadi et al. 2011).

Los mayores porcentajes de lípidos encontrados en la diatomea cuando fue cultivada con Humus puede atribuirse al aumento de la irradiancia, que junto a la presencia mayoritaria de nitrógeno amoniacal, pueden crear un ambiente de estrés, promoviendo la síntesis de esta macromolécula (McGinnis et al. 1997, Solovchenko et al. 2008), sirviendo como fuente primordial de energía ante condiciones estresantes (Fábregas et al. 2004). Por otro lado, tanto el contenido de lípidos totales, así como la modificación en la calidad de los mismos se ve influenciada por la intensidad de luz. Algunas especies como Phaeodactylum tricornutum, Pavlova sp. y Nannochloropsis spp. son conocidas como grandes productoras de AEP a bajas irradiancias (Khotimchenko \& Yakovleva 2005, Patil et al. 2007), mientras que Skeletonema costatum ha sido conocida como poca productora de AEP a bajas intensidades de luz (Guihéneuf et al. 2009).

Pratoomyot et al. (2005) reportan en Thalassiosira sp. como principales ácidos grasos al 16:0; 16:1 n-7 y 20:5 n-3 con bajos porcentajes del 22:6 n-3 cuando fue cultivada a $143 \mu \mathrm{E} /$ $\mathrm{m}^{2}$.s. Un perfil similar fue encontrado en esta investigación, aunque los máximos de AEP $(6.20 \%)$ se obtuvieron a $60 \mu \mathrm{E} / \mathrm{m}^{2}$.s en Algal, evidenciándose una tendencia a su disminución, con el incremento de la irradiancia en ambos medios, mientras que las concentraciones de $\mathrm{ADH}$ apenas fueron detectadas. Este comportamiento permite sugerir sobre el incremento en los porcentajes de ácidos grasos saturados como consecuencia en la disminución de los AGPs por efectos del aumento en la irradiancia de los cultivos.

Basova (2005) menciona que los cambios en los porcentajes de ácidos grasos microalgales están relacionados con las proporciones de las diferentes clases de lípidos los cuales pueden variar entre 2 y $44 \%$ dependiendo de la especie y las condiciones de cultivo. En este estudio, se observan mayores porcentajes de AGPs a baja irradiancia en ambos medios y principalmente AEP, el cual pudiera estar asociado con los galactolípidos, considerados componentes mayoritarios en membranas tilacoidales para preservar la fluidez membranal y el transporte de electrones en la fotosíntesis (Mock \& Kroon 2002).

La disminución en el contenido de clorofila $a$ como consecuencia del incremento en el nivel de irradiancia sobre cultivos microalgales se ha descrito previamente (Miskiewicz et al. 2002, Carvalho et al. 2009), esto coincide con los resultados evidenciados en esta investigación. Masuda et al. (2003) indicaron que bajas irradiancias, inducen en las células el incremento de pigmentos fotosintéticos tales como clorofila $a$ a fin de aumentar su capacidad para captación de luz (Mock \& Kroon 2002) y optimizar la eficiencia del fotosistema dos (PSII) (Janknegt et al. 2008).

En el caso de los carotenoides totales, su incremento en conjunto con la irradiancia han sido atribuidos a la síntesis de pigmentos fotoprotectores como las xantofilas, diadinoxantinas y diatoxantinas los cuales juegan un rol fundamental en la prevención de daños al aparato fotosintético de diatomeas expuestas a elevadas irradiancias (Lavaud et al. 2002).

Los resultados demuestran que la cepa de T. pseudonana analizada presenta un margen amplio de adaptación, evidenciados por los patrones diferentes de comportamiento, producto de las perturbaciones fisiológicas ocasionadas por cambios en la irradiancia y en las proporciones de nutrientes presentes en ambos medios de cultivo. 


\section{AGRADECIMIENTOS}

Los autores expresan su agradecimiento al Consejo de Investigación de la Universidad de Oriente, por su financiamiento a través del proyecto CI.-2-030603-1282/06. Se agradece el apoyo brindado para los análisis de los ácidos grasos a: Elena Palacios, Olivia Arjona y Laura Carreón del CIBNOR, México.

\section{RESUMEN}

Thalassiosira pseudonana es utilizada como alimento en acuicultura, pero su valor nutricional está influenciado por las condiciones de crecimiento. Sistemas semicontinuos, en fase de estabilización, con tres irradiancias $\left(60,120\right.$ y $180 \mu \mathrm{E} / \mathrm{m}^{2}$.s) y dos medios de cultivo (Algal y Humus) fueron las condiciones en las que se determinó el crecimiento y componentes bioquímicos de T. pseudonana. En Humus a $180 \mu \mathrm{E} / \mathrm{m}^{2}$.s se obtuvieron las máximas densidades celulares (entre 3.86 y $4.62 \times 10^{6} \mathrm{cel} / \mathrm{mL}$ ) y mayores concentraciones de lípidos y carbohidratos, con porcentajes de $20.3 \pm 2.28$ y $16.6 \pm 2.43$, respectivamente. A $180 \mu \mathrm{E} / \mathrm{m}^{2}$.s en Algal se observaron los mayores valores de proteínas $(45.0 \pm 5.05)$ y carotenoides totales $(0.5 \pm 0.01)$. El contenido de clorofila $a$ fue favorecido por baja intensidad de luz, principalmente en Algal, con máximos de $0.9 \pm 0.04 \%$. El grado de insaturación de los ácidos grasos disminuyó por incremento de la irradiancia en ambos medios, estando mayoritariamente representados por el ácido eicosapentaenoico, 20:5 n-3 (AEP), con porcentajes máximos (6.20\%) en Algal a $60 \mu \mathrm{E} / \mathrm{m}^{2}$.s. Los resultados muestran múltiples respuestas fisiológicas de T. pseudonana frente a cambios en las condiciones de crecimiento, las cuales pueden ser aprovechadas para mejorar su valor nutricional como alimento de organismos cultivados, utilizando medios de cultivo alternativos, que disminuyan los costos en la producción microalgal.

Palabras clave: Thalassiosira pseudonana, acuicultura tropical, ácidos grasos, autóctona, cultivo semicontinuo.

\section{REFERENCIAS}

Basova, M. 2005. Fatty acid composition of lipids in microalgae. Algología 15: 188-213.

Bligh, E. \& W. Dyer. 1959. A rapid method of total lipid extraction and purification. Can. J. Biochem. Physiol 37: 911-917.

Carvalho, A.P., C.M. Monteiro \& F. Malcata. 2009. Simultaneous effect of irradiance and temperature on biochemical composition of microalga Pavlova lutheri. J. Appl. Phycol 21: 543-552.
Chaneva, G., S. Furnadzhieva, K. Minkova \& J. Lukavsky. 2007. Effect of light and temperature on the cyanobacterium Arthronema africanum a prospective phycobiliprotein-producing strain. J. Appl. Phycol. 19: 537-544.

De la Peña, R. \& C. Villegas. 2005. Cell growth, effect of filtrate and nutritive value of the tropical Prasinophyte Tetraselmis tetrathele (Butcher) at different phases of culture. Aquaculture Res. 36: 1500-1508.

Dubois, M., K. Gilles, J. Halmilton, P. Rebers \& F. Smith. 1956. Colorimetric method for determination of sugars and related sustances. Anal. Chem. 2: 350-356.

Fábregas, J., J. Abalde, C. Herrero, B. Cabezas \& M. Veiga. 1984. Growth of the marine microalgae Tetraselmis suecica in batch cultures with different salinities and nutrient concentrations. Aquaculture 42: 207-215.

Fábregas, J., M. Patiño, B. Arredondo-Vega, J. Tobar \& A. Otero. 1995. Renewal rate and nutrient concentration as tools to modify productivity and biochemical composition of cyclostat cultures of the marine microalga Dunaliella tertiolecta. Appl. Microbiol. Biotechnol. 44: 287-292.

Fábregas, J., A. Dominguez, M. Regueiro, A. Maseda \& A. Otero. 2000. Optimization of culture medium for the continuous cultivation of the microalga Haematococcus pluvialis. Appl. Microbiol. Biotechnol. 53: 530-535.

Fábregas, J., A. Maseda, A. Dominguez \& A. Otero. 2004. The cell composition of Nannochloropsis sp. Changes of different irradiances in semicontinuous culture. World J. Microbiol. Biotechnol. 20: 31-35.

Guihéneuf, F., V. Mimouni, L. Ulmann \& G. Tremblin. 2009. Combined effects of irradiance level and carbon source on fatty acid and lipid class composition in the microalga Pavlova lutheri commonly used in mariculture. J. Exp. Mar. Biol. Ecol. 369: 136-143.

Guzmán-Murillo, M., C. López-Bolaños, T. LedesmaVerdejo, G. Roldan-Libenson, M. Cadena-Roa \& F. Ascencio. 2007. Effects of fertilizer-based culture media on the production of exocellular polysaccharides and cellular superoxide dismutase by Phaeodactylum tricornutum (Bohlin). J. Appl. Phycol. 19: 33-41.

Hou, J.J, B.Q. Huang, Z.R Cao, J.X. Chen \& H.S. Hong. 2007. Effects of nutrient limitation on pigments in Thalassiosira weissflogii and Prorocentrum donghaiense. J. Integr. Plant Biol. 49: 86-697.

Janknegt, P.J., W.H. Van de Poll \& R.J.W. Visser. 2008. Oxidative stress responses in the marine Antarctic diatom Chaetoceros brevis (Bacillariophyceae) during photoacclimation. J. Phycol. 44: 957-966.

Khotimchenko, S.V. \& I.M. Yakovleva. 2005. Lipid composition of the red alga Tichocarpus crinitus exposed to 
different levels of photon irradiance. Phytochemistry 66: 73-79.

Kiatmetha, P., W. Siangdang, B. Bunnag, S. Senapin \& B. Withyachumnarnkul. 2011. Enhancement of survival and metamorphosis rates of Penaeus monodon larvae by feeding with the diatom Thalassiosira weissflogii. Aquaculture Int. 19: 599-609.

Lavaud, J., B. Rousseau, V. Gorkom \& A.L. Etienne. 2002. Influence of the diadinoxanthin pool size on photoprotection in the marine planktonic diatom Phaeodactylum tricornutum. Plant Physiol. 129: 1398-1406.

Liu, S., Z. Guo, T. Li, H. Huang \& S. Lin. 2011. Photosynthetic efficiency, cell volume, and elemental stoichiometric ratios in Thalassirosira weissflogii under phosphorus limitation. Chin. J. Oceanol. Limnol. 29: 1048-1056.

Lowry, O., H. Rosebrough, A. Farr \& R. Randall. 1951. Protein measurement with the folin-phenol reagent. J. Biol. Chem. 193: 265-275.

Madigan, M., J. Martinko \& J. Parker. 1999. Brock: Biología de los microorganismos. Prentice May, Madrid, España.

Mansour, M., D. Frampton, P. Nichols \& J. Volkman. 2005. Lipid and fatty acid yield of nine stationaryphase microalgae: Applications and unusual $\mathrm{C}_{24}$ $\mathrm{C}_{28}$ polyunsaturated fatty acids. J. Appl. Phycol. 17: 287-300.

Marsh, J. \& D. Weinstein. 1966. Simple charring method for determination of lipids. J. Lipids Res. 7: 574-592.

Masuda, T., A. Tanaka \& A. Melis. 2003. Chlorophyll antenna size adjustments by irradiance in Dunaliella salina involve coordinate regulation of chlorophyll $a$ oxygenase $(C A O)$ and $L h c b$ gene expression. Plant Mol. Biol. 51: 757-771.

McGinnis, K.M., T.A. Dempster \& M.R. Sommerfeld. 1997. Characterization of the growth and lipid content of the diatom Chaetoceros muelleri. J. Appl. Phycol. 9: 19-24.

Meseck, S.L., J.H. Alix \& G.H. Wikfors. 2005. Photoperiod and light intensity effects on growth and utilization of nutrients by the aquaculture feed microalga, Tetraselmis chui (PLY429). Aquaculture 246: 393-404.

Mischke, C.C. \& P.V. Zimba. 2004. Plankton community responses in earthen channel catfish nursery ponds under various verfilization regimes. Aquaculture 233: 219-235.

Miskiewicz, E., A.G. Ivanov \& N.P. Huner. 2002. Stoichiometry of the photosynthetic apparatus and phycobilisome structure of the cyanobacterium Plectonema boryanum UTEX 485 are regulated by both light and temperature. Plant Physiol. 130: 1414-1425.
Mock, T. \& B.M.A. Kroon. 2002. Photosynthetic energy conversion under extreme conditions - II: the significance of lipids under light limited growth in Antarctic sea ice diatoms. Phytochemistry 61: 53-60.

Otero, A., D. García, E. Morales, J. Arán \& J. Fábregas. 1997. Manipulation of the biochemical composition of the eicosapentaenoic acid-rich microalga Isochrysis galbana in semicontinuous cultures. Biotechnol. Appl. Biochem. 26: 171-177.

Patil, V., T. Källqvist, E. Olsen, G. Vogt \& H. Gislerod. 2007. Fatty acid composition of 12 microalgae for possible use in aquaculture feed. Aquacult. Int. 15: $1-9$.

Pratoomyot, J., P. Srivilas \& T. Noiraksar. 2005. Fatty acids composition of 10 microalgal species. Songklanakarin J. Sci. Technol. 27: 1179-1187.

Priyadarshani, I., D. Sahu \& B. Rath. 2012. Alga in aquaculture. Int. J. Health Sci. Res. 2: 108-114.

Roberts, S., K. Shelly \& J. Beardall. 2008. Interactions among phosphate uptake, photosynthesis and chlorophyll fluorescence in nutrient-limited cultures of the Chlorophyte microalga Dunaliella tertiolecta. J. Phycol. 44: 662-669.

Rosen, B.H. \& R.C. Lowe. 1984. Physiological and ultrastructural responses of Cyclotella meneghiniana (Bacillariophyta) to ligth intensity and nutrient limitation. J. Phycol. 20: 173-182.

Sánchez-Saavedra, M. \& D. Voltolina. 2002. Effects of photon fluence rates of white and blue-green light on growth efficiency and pigment content of three diatom species in batch culture. Ciencias Mar. 28: 273-279.

Sandnes, J.M., T. Källqvist, D. Wenner \& H. Gislerod. 2005. Combined influence of light and temperature on growth rates of Nannochloropsis oceanica: linking cellular responses to large-scale biomass production. J. Appl. Phycol. 17: 515-525.

Sato, N. \& N. Murata. 1988. Membrane lipids. Meth. Enzymol. 167: 251-259.

Seyfabadi, J., Z. Ramezanpour \& Z.A. Khoeyi. 2011. Protein, fatty acid, and pigment content of Chlorella vulgaris under different light regimes. J. Appl. Phycol. 23: 721-726.

Shaw, P., G. Jones, D. Smith \& R. Johns. 1989. Intraspecific variations in the fatty acids of the diatom Skeletonema costatum. Phytochemistry 28: 811-815.

Sokal, R. \& F. Rohlf. 1995. Biometry. W.H. Freeman and Co., Nueva York, EE.UU.

Solovchenko, A.E., M.N. Merzlyak, O.B. Chivkunova, I.V. Reshetnikova, I. Khozina-Goldberg, S. Didi-Cohen \& Z. Cohen. 2008. Effects of illumination and nitrogen starvation on accumulation of arachidonic acid by the 
microalga. Parietochloris incisa. Moscow Univ. Biol. Sci. Bull. 63: 44-48.

Strickland, J. \& T. Parsons. 1972. A practical handbook of sea water analysis. Fisheries Research Board of Canada. Bulletin 167, Ottawa, Canadá.

Uriarte, I., R. Roberts \& A. Farias. 2006. The effect of nitrate supplementation on the biochemical composition of benthic diatoms and the growth and survival of post-larval abalone. Aquaculture 261: 423-429.

Valenzuela-Espinoza, E., F. Lafarga-De la Cruz, R. MillánNuñez \& F. Nuñez-Cebrero. 2005. Crecimiento, consumo de nutrientes y composición proximal de Rhodomonas sp. cultivada con medio $\mathrm{f} / 2 \mathrm{y}$ fertilizantes agrícolas. Ciencias Mar. 31: 79-89.
Vílchez, C., E. Forján, M. Cuaresma, F. Bédmar, I. Garbayo \& J.M. Vega. 2011. Marine carotenoids: biological functions and commercial applications. Mar. Drugs 9: 319-333.

Wood, B.J., P.H. Grimson, J.B. German \& M. Turner. 1999. Photoheterotrophy in the production of phytoplankton organisms. J. Biotechnol. 70: 175-183.

Young, E.B. \& J. Beardall. 2003. Rapid ammonium and nitrate induced perturbations to chlorophyll $a$ fluorescence in nitrogen-stressed Dunaliella tertiolecta (Chlorophyta). J. Phycol. 39: 332-42.

Zhukova, N.V. 2004. Changes in the lipid composition of Thalassiosira pseudonana during its life cycle. Russ. J. Plant Physiol. 51: 702-707. 
